\title{
Fe de errores: Recomendaciones para el estudio y tratamiento de la anemia en pacientes con enfermedad renal crónica: Actualización mayo 2017 [Nefrol Latinoam. 2017;14(3):85-116]
}

\author{
Erratum: Recommendations for the study and treatment of anaemia in patients with \\ chronic kidney disease. Update May 2017 [Nefrol Latinoam. 2017;14(3):85-116]
}

Raul G. Carlini ${ }^{*}$, María N. Campistrús², Liliana Andrade 3 , Carlos Blanco ${ }^{4}$, Liliana Chifflet ${ }^{5}$, Zulma C. Cruz de Trujillo ${ }^{6}$, María E. Fernandes-Canziani', Thais A. Forster ${ }^{8}$, Marcus Gomes-Bastos ${ }^{9}$, Gregorio T. Obrador ${ }^{10}$, Jorge F. D. Pérez-Oliva ${ }^{11}$, Hugo Poblete-Badal'12 y Fernando Renjel-Claros ${ }^{13}$

${ }^{1}$ Co-coordinador, Hospital Universitario de Caracas, Universidad Central de Venezuela, Caracas, Venezuela; ${ }^{2}$ Co-coordinadora, Comité de Educación Médica Continua, Sociedad Uruguaya de Nefrología, Montevideo, Uruguay; ${ }^{3}$ Hospital Churruca Visca (PFA), Buenos Aires, Argentina; ${ }^{4}$ Servicio de Nefrología, Hospital Aeronáutico Central, Buenos Aires, Argentina; ${ }^{5}$ Fondo Nacional de Recursos, Montevideo, Uruguay; ${ }^{6}$ Facultad de Medicina, Universidad de El Salvador, San Salvador, El Salvador; ${ }^{7}$ Disciplina de Nefrología, Universidade Federal de São Paulo, São Paulo, Brasil; ${ }^{8}$ Ciencias de Información en Salud, Montevideo, Uruguay; ${ }^{9}$ Serviço de Nefrologia, Hospital Universitário da Universidade Federal de Juiz de Fora, Juiz de Fora, Brasil; ${ }^{10}$ Escuela de Medicina, Universidad Panamericana, Ciudad de México, México; ${ }^{11}$ Instituto Nacional de Nefrología "Dr. Abelardo Buch

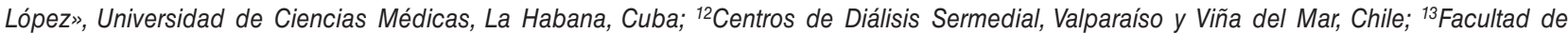
Medicina, Universidad Mayor de San Simón, Cochabamba, Bolivia

En el artículo publicado anteriormente, «Recomendaciones para el estudio y tratamiento de la anemia en pacientes con enfermedad renal crónica: Actualización mayo 2017», [Nefrol Latinoam. 2017;14(3):85-116] se ha detectado un error:

- En el capítulo 4, apartado 19 (página 91) debe decir $\mathrm{Hb}<10 \mathrm{~g} / \mathrm{dl}$ (en vez de $\mathrm{Hb}>10 \mathrm{~g} / \mathrm{dl}$ ).

- En el capítulo 4, apartado 19 (página 104) debe decir $\mathrm{Hb}<10 \mathrm{~g} / \mathrm{dl}$ (en vez de $\mathrm{Hb}>10 \mathrm{~g} / \mathrm{dl}$ ).

- En el capítulo 3 (página 102), al comienzo de la segunda columna hay un símbolo que está errado.
Debe decir $\mathbf{H b}$ objetivo > $\mathbf{1 2} \mathbf{~ g / d l}$ (en vez de $\mathrm{Hb}$ objetivo $\square \mathrm{Hb} 12 \mathrm{~g} / \mathrm{dl})$.

- En el capítulo 6 (página 92) punto 32.6 debe decir Hiperparatiroidismo secundario severo (en vez de Hiperperpartiroidismo severo).

- En la tabla 3 (página 93) debe decir Hiperparatiroidismo secundario severo (en vez de Hiperparatiroidismo severo).

Véase contenido relacionado en DOI: https://doi.org/10.1016/j.nefrol.2017.09.002

\section{Correspondencia:}

${ }^{*}$ Raul G. Carlini

E-mail: raul.carlini@gmail.com DOI: 10.24875/NEFRO.M18000003
Disponible en internet: 19-10-2018

Nefro Latinoam. 2018;15:65 www.nefrologialatinoamericana.com 2444-9032/@ 2018 Sociedad Latinoamericana de Nefrología e Hipertensión. Publicado por Permanyer México SA de CV. Este es un artículo Open Access bajo la licencia CC BY-NC-ND (http://creativecommons.org/licenses/by-nc-nd/4.0/). 